\title{
EFeito de Herbicidas sobre Quatro Espécies de Trapoeraba ${ }^{1}$
}

\author{
Effect of Herbicides on Four Wandering-Jew Species
}

\begin{abstract}
ROCHA, D.C. ${ }^{2}$, RODELLA, R.A. ${ }^{3}$, MARTINS, D. ${ }^{4}$ e MACIEL, C.D.G. ${ }^{5}$
RESUMO - O controle químico de espécies do gênero Commelina (trapoerabas) é, muitas vezes, insatisfatório, apesar do uso intenso de herbicidas. O presente trabalho teve como objetivo avaliar o controle de quatro espécies daninhas de Commelina com o uso de diferentes herbicidas aplicados em pós-emergência. Foram avaliadas plantas de C. benghalensis, C. villosa, C. diffusa e C. erecta, em estádio com mais de quatro folhas e caules com cerca de 15 a $25 \mathrm{~cm}$ de comprimento, submetidas aos tratamentos com carfentrazone-ethyl (30 e $\left.50 \mathrm{~g} \mathrm{ha}^{-1}\right)$, glyphosate $\left(960 \mathrm{~g} \mathrm{ha}^{-1}\right)$, carfentrazone-ethyl + glyphosate $\left(30+960 \mathrm{~g} \mathrm{ha}^{-1}\right) \mathrm{e}$ sulfentrazone + glyphosate $\left(150+960 \mathrm{~g} \mathrm{ha}^{-1}\right)$, além de uma testemunha sem aplicação. $\mathrm{O}$ delineamento experimental foi inteiramente casualizado, com quatro repetições. Verificouse que o controle químico de trapoerabas foi dependente da espécie, sendo C. benghalensis controlada mais eficientemente com os tratamentos testados, enquanto $C$. erecta apresentou o menor controle. A aplicação de glyphosate em mistura com carfentrazone-ethyl foi mais eficiente no controle das espécies de trapoeraba do que os demais tratamentos empregados.
\end{abstract}

Palavras-chave: Commelina benghalensis, Commelina diffusa, Commelina erecta, Commelina villosa, controle químico.

\begin{abstract}
Chemical weed control of Commelina species (wandering-jew) is sometimes unsatisfactory. This work was carried out to observe the effect of herbicides on Commelina species at post-emergence. C. benghalensis, C. villosa, C. diffusa and C. erecta were treated with carfentrazone-ethyl (30 $\mathrm{g} \mathrm{ha}^{-1}$ and $\left.50 \mathrm{~g} \mathrm{ha}^{-1}\right)$, glyphosate $\left(960 \mathrm{~g} \mathrm{ha}^{-1}\right)$, carfentrazone-ethyl plus glyphosate $\left(30+960 \mathrm{~g} \mathrm{ha}^{-1}\right)$, sulfentrazone plus glyphosate $\left(150+960 \mathrm{~g} \mathrm{ha}^{-1}\right)$, and a control, when the plants displayed 15 to $25 \mathrm{~cm}$ long stems. The experimental design was completely randomized with four replications. Commelina chemical control was different among species. Best control was achieved with $C$. benghalensis and $\mathbf{C}$. erecta had the worst level of control. The treatment with carfentrazone-ethyl plus glyphosate showed the best results on the control of these four Commelina species, compared to other applications.
\end{abstract}

Keywords: Commelina benghalensis, Commelina diffusa, Commelina erecta, Commelina villosa, chemical control.

\section{INTRODUÇÃO}

Das plantas da família Commelinaceae, as do gênero Commelina têm o maior número de representantes no Brasil, sendo $C$. benghalensis, C. diffusa e C. erecta espécies muito difundidas em todo o território nacional (Barreto, 1997). Por suavez, C. villosa foi primeiramente registra- da no Distrito Federal e nos Estados de Goiás e da Bahia, tendo sido já encontrada no Paraná (Rocha et al., 2000; Penckowski \& Rocha, 2006). O glyphosate é recomendado para controle de diversas plantas daninhas, mas, no caso de trapoerabas (Commelina spp.), muitas vezes não ocorre efeito satisfatório. Para C. benghalensis, Lorenzi (2006) apresenta uma listagem de

1 Recebido para publicação em 19.12.2006 e na forma revisada em 17.4.2007.

2 Professora Adjunta do Dep. de Biologia, Setor de Ciências Biológicas e da Saúde, UEPG, 84030-900 Ponta Grossa-PR, <dalva_rocha@uol.com.br>; ${ }^{3}$ Professor Doutor do Dep. de Botânica, Instituto de Biociências de Botucatu, UNESP, 18618-000 Botucatu-SP, <rodella@ibb.unesp.br>; ${ }^{4}$ Professor Adjunto do Dep. de Produção Vegetal, Faculdade de Ciências Agronômicas, UNESP, 18603-970 Botucatu-SP, <dmartins@fca.unesp.br>; ${ }^{5}$ Professor Doutor da Escola Superior de Agronomia de Paraguaçu Paulista, 19700-000 Paraguaçu Paulista-SP, <macielconsultoria@ hotmail.com>. 
88 herbicidas, dos quais somente 33 formulações inibem o desenvolvimento dessa planta daninha em menos de $85 \%$.

Diversos trabalhos foram realizados usando glyphosate em misturas, com a finalidade de obter controle satisfatório. Tollervey et al. (1979) indicaram a mistura glyphosate $+2,4-\mathrm{D}$ para controlar trapoeraba em diversas culturas, provavelmente devido ao sinergismo entre os produtos. Para controlar essa mesma planta daninha, especificamente na cultura de cacau, Carvalho et al. (1991) utilizaram glyphosate e 2,4-D aplicados de forma isolada em três diferentes doses e na forma de mistura, porém não obtiveram controle satisfatório. Além da mistura com 2,4-D, a eficiência de glyphosate em mistura com outros produtos, como diuron, $\mathrm{KCl}$ e uréia, foi testada por Galli (1991) para controlar C. virginica em pomares de citros, constatando-se melhor resultado com a mistura de glyphosate $+2,4-\mathrm{D}$ aos 28 dias após a aplicação dessa formulação, embora o controle total das plantas não tenha sido alcançado. Ramos \& Durigan (1996) utilizaram glyphosate e 2,4-D amina, aplicados isoladamente ou em mistura, e também constataram que a aplicação de glyphosate na forma de mistura apresentou o melhor controle de $C$. virginica em citros. A mistura de carfentrazone-ethyl com glyphosate proporcionou efeito aditivo no controle de C. benghalensis, como verificaram Werlang \& Silva (2002).

Algumas hipóteses já foram propostas para explicar essa diferença de controle de um tratamento para o outro. Segundo Wilson (1981), a dificuldade de controle de espécies da familia Commelinaceae pode ser atribuída ao duplo mecanismo de reprodução que elas apresentam: por sementes e por enraizamento dos nós. De La Vega et al. (2000) mencionaram a dificuldade de controle de três espécies de trapoerabas, mas somente fizeram experimentos com C. erecta e concluiram que o efeito do glyphosate sobre as plantas depende da fase de desenvolvimento.

O presente trabalho teve como objetivo avaliar o efeito de diferentes herbicidas, aplicados em pós-emergência, sobre as espécies daninhas C. benghalensis, C. villosa, C. diffusa e C. erecta.

\section{MATERIAL E MÉTODOS}

Foram utilizadas quatro espécies de trapoeraba: Commelina benghalensis, Commelina villosa, Commelina diffusa e Commelina erecta. As plantas foram cultivadas em vasos com capacidade de um litro, contendo solo e mistura de substrato na proporção $7: 3(\mathrm{v} / \mathrm{v})$, os quais foram mantidos em casa de vegetação pertencente ao Departamento de Botânica do Instituto de Biociências de Botucatu - UNESP, sendo semanalmente alternados de posição para fins de casualização. Realizou-se adubação com 2,5 g da fórmula 4-14-8 de NPK no início e a intervalos regulares de 15 dias. As características químicas da mistura de solo e substrato estão apresentadas na Tabela 1. Essas plantas foram submetidas a seis tratamentos: 1) testemunha; 2) carfentrazone-ethyl (30 $\left.\mathrm{g} \mathrm{ha}^{-1}\right)$; 3 ) carfentrazone-ethyl (50 $\left.\mathrm{g} \mathrm{ha}^{-1}\right)$; 4) carfentrazoneethyl + glyphosate $\left.\left(30+960 \mathrm{~g} \mathrm{ha}^{-1}\right) ; 5\right)$ sulfentrazone + glyphosate $\left(150+960 \mathrm{~g} \mathrm{ha}^{1}\right)$; e 6) glyphosate (960 $\left.\mathrm{g} \mathrm{ha}^{-1}\right)$.

No momento da pulverização dos herbicidas, as plantas se caracterizavam por apresentar caules de comprimento entre 15 e $25 \mathrm{~cm}$ e com mais de quatro folhas. Os herbicidas foram aplicados em duas etapas, utilizandose um pulverizador costal pressurizado a $\mathrm{CO}_{2}$ $\left(2 \mathrm{kgf} \mathrm{m}^{-3}\right)$ e acoplado a uma barra contendo quatro bicos de jato plano $110.02 \mathrm{VS}$, com consumo de calda equivalente a $200 \mathrm{~L} \mathrm{ha}^{-1}$. Na primeira etapa, os herbicidas foram aplicados sobre plantas de $C$. benghalensis e $C$. villosa e, na segunda, sobre plantas de $C$. diffusa e C. erecta. Em ambas as etapas não havia vento e a temperatura ambiente era de $26^{\circ} \mathrm{C}$.

A avaliação do efeito causado pelos herbicidas às plantas de Commelina foi realizada com base na visualização dos sintomas de toxicidade apresentados pelas plantas durante 28 dias após a aplicação (DAA). Os sintomas observados foram: quantidade de folhas mortas, massa seca acumulada, inibição do crescimento, quantidade e uniformidade das injúrias e capacidade de rebrota das plantas. Foi utilizada uma escala percentual de controle, comparando as testemunhas com as plantas tratadas com herbicidas, em que $0 \%$ correspondia a nenhum sintoma observado e $100 \%$ indicava morte das plantas (SBCPD, 1995). Após a última avaliação, as plantas foram submetidas à 
Tabela 1 - Resultado da análise química da mistura de solo e substrato utilizada no cultivo das espécies daninhas de trapoeraba

\begin{tabular}{|c|c|c|c|c|c|c|c|c|c|}
\hline $\begin{array}{c}\mathrm{pH} \\
\left(\mathrm{CaCl}_{2}\right)\end{array}$ & MO & $\mathrm{P}_{\text {resina }}$ & $\mathrm{H}+\mathrm{Al}$ & K & $\mathrm{Ca}$ & $\mathrm{Mg}$ & SB & CTC & $\mathrm{V}$ \\
\hline & $--\mathrm{g} \mathrm{kg}^{-1}--$ & $--\mathrm{mg} \mathrm{dm}^{-3}--$ & \multicolumn{6}{|c|}{ - $\mathrm{mmol}_{\mathrm{c}} \mathrm{dm}^{-3}$} & --- \% --- \\
\hline 5,06 & 24,30 & 4,70 & 38,60 & 2,80 & 28,60 & 11,60 & 43,00 & 81,60 & 53,00 \\
\hline
\end{tabular}

secagem em estufa de circulação forçada de ar com temperatura variável entre 65 e $70^{\circ} \mathrm{C}$, até atingirem massa constante, e em seguida pesadas.

Os dados foram submetidos à análise de variância, e as médias, comparadas pelo teste $\mathrm{t}$ (Pimentel-Gomes, 1976).

\section{RESULTADOS E DISCUSSÃO}

O efeito dos herbicidas sobre C. benghalensis, C. villosa, C. diffusa e C. erecta está expresso em porcentagem de controle nas Tabelas 2, 3, 4 e 5 , respectivamente.

No primeiro dia após a aplicação de carfentrazone-ethyl, na forma isolada ou em mistura com glyphosate, as plantas de $C$. benghalensis apresentaram sintomas severos, que levaram à morte delas aos 14 DAA (Tabela 2), ou seja, seus ramos secundários foram danificados em diversas regiões, caracterizadas por clorose acentuada, estrangulamento nos entrenós e exsudação de seiva, enquanto suas folhas mais jovens apresentaram-se enroladas e murchas, acentuadamente necrosadas e amarelecidas.

Por sua vez, os sintomas observados em plantas de C. villosa submetidas a esses tratamentos com carfentrazone-ethyl foram menos expressivos que aqueles observados em C. benghalensis; portanto, esses tratamentos apresentaram resultados menos eficazes no controle de C. villosa (Tabela 3). Nesse caso, observou-se que os ramos primários se mantiveram sem estrangulamentos na região dos entrenós e sem exsudação de seiva até o terceiro dia de avaliação, embora, no primeiro dia, também tenham sido notados necrose acentuada, enrolamento e murchamento das folhas, principalmente dos ramos superiores, o que pode ser caracterizado como efeito "guardachuva", ou seja, retenção de calda de pulverização no estrato superior de folhas em relação ao estrato inferior. Os melhores resultados no controle de $C$. villosa com carfentrazone-ethyl foram obtidos quando o herbicida foi aplicado na forma isolada, na dose de $50 \mathrm{~g} \mathrm{ha}^{-1}$, e também na mistura de carfentrazone-ethyl + glyphosate $\left(30+960 \mathrm{~g} \mathrm{ha}^{-1}\right)$, como mostra a Tabela 3.

As espécies C. diffusa e C. erecta tiveram seus desenvolvimentos inibidos apenas parcialmente sob a ação de carfentrazone-ethyl nos três tratamentos testados, não sendo, conseqüentemente, controladas de forma eficiente por esse herbicida (Tabelas 4 e 5 ). Os tratamentos contendo carfentrazone-ethyl provocaram nas plantas de $C$. diffusae de $C$. erecta sintomas ainda menos severos que os relatados para C. villosa. Não foi observada exsudação de seiva ou clorose dos ramos, mas notou-se o murchamento geral das plantas, que apresentavam estrangulamento somente nos entrenós terminais do ramo, leve clorose de algumas folhas e necrose apenas nas folhas mais jovens dos ponteiros com o enrolamento das suas margens.

O tratamento com glyphosate aplicado na forma isolada também não foi capaz de inibir completamente o desenvolvimento das plantas de C. benghalensis, C. diffusa e C. erecta(Tabelas 2, 4 e 5), porém esse tratamento foi capaz de controlar de forma satisfatória as plantas de C. villosa (Tabela 3). Monquero et al. (2005) também constataram que plantas de $C$. benghalensis foram tolerantes à aplicação de glyphosate. $\mathrm{O}$ único sintoma visualizado decorrente da aplicação de glyphosate na forma isolada, em todas as espécies de Commelina estudadas, foi o amarelecimento de folhas, sendo tardiamente observado em relação aos demais tratamentos. Tuffi Santos et al. (2004) observaram também 
Tabela 2 - Efeito de diferentes herbicidas sobre Commelina benghalensis, aplicados em pós-emergência tardia. Botucatu-SP

\begin{tabular}{|c|c|c|c|c|c|c|c|c|c|}
\hline \multirow[t]{2}{*}{ Tratamento } & \multirow{2}{*}{$\begin{array}{c}\text { Dose } \\
\left(\mathrm{g} \mathrm{ha}^{-1}\right)\end{array}$} & \multicolumn{7}{|c|}{$\begin{array}{c}\text { Controle (\%) } \\
\text { Dias após a Aplicação }\end{array}$} & \multirow{2}{*}{$\begin{array}{c}\text { Massa } \\
\text { Seca } \\
(\mathrm{g})\end{array}$} \\
\hline & & 1 & 3 & 6 & 9 & 14 & 21 & 28 & \\
\hline Testemunha & - & $0,0 \mathrm{~d}$ & $0,0 \mathrm{c}$ & $0,0 \mathrm{~d}$ & $0,0 \mathrm{~d}$ & $0,0 \mathrm{~d}$ & $0,0 \mathrm{~d}$ & $0,0 \mathrm{c}$ & $13,4 \mathrm{a}$ \\
\hline Carfentrazone $\mathrm{e}^{\mathrm{L}}$ & 30 & $85,0 \mathrm{ab}$ & $91,7 \mathrm{a}$ & $97,5 \mathrm{a}$ & $98,5 \mathrm{a}$ & $100,0 \mathrm{a}$ & $100,0 \mathrm{a}$ & $100,0 \mathrm{a}$ & $0,0 \mathrm{c}$ \\
\hline Carfentrazone & 50 & $76,7 b$ & $91,2 \mathrm{a}$ & $98,7 \mathrm{a}$ & $99,2 \mathrm{a}$ & $100,0 \mathrm{a}$ & $100,0 \mathrm{a}$ & $100,0 \mathrm{a}$ & $0,0 \mathrm{c}$ \\
\hline Carfentrazone+glyphosate & $30+960$ & $90,0 \mathrm{a}$ & $94,2 \mathrm{a}$ & $100,0 \mathrm{a}$ & $100,0 \mathrm{a}$ & $100,0 \mathrm{a}$ & $100,0 \mathrm{a}$ & $100,0 \mathrm{a}$ & $0,0 \mathrm{c}$ \\
\hline Sulfentrazone+glyphosate & $150+960$ & $50,0 \mathrm{c}$ & $61,2 b$ & $57,5 \mathrm{~b}$ & $90,0 \mathrm{~b}$ & $80,2 \mathrm{~b}$ & $73,2 \mathrm{~b}$ & $28,7 \mathrm{~b}$ & $1,0 \mathrm{c}$ \\
\hline Glyphosate & 960 & $1,7 \mathrm{~d}$ & $3,5 \mathrm{c}$ & $6,5 \mathrm{c}$ & $13,7 \mathrm{c}$ & $23,2 \mathrm{c}$ & $16,2 \mathrm{c}$ & $23,7 \mathrm{bc}$ & $4,3 \mathrm{~b}$ \\
\hline $\mathrm{F}$ & & $73,04 * *$ & $187,04 * *$ & 482,25 ** & $1175,00^{* * *}$ & $184,24 * *$ & $131,42^{* * *}$ & $15,96^{* *}$ & $57,52 * *$ \\
\hline $\mathrm{CV}(\%)$ & & 18,92 & 11,42 & 7,11 & 4,09 & 9,72 & 12,22 & 39,39 & 44,81 \\
\hline d.m.s. & & 11,74 & 7,98 & 5,23 & 3,35 & 8,02 & 9,73 & 28,37 & 1,72 \\
\hline
\end{tabular}

** significativo a $1 \%$ de probabilidade.

Médias seguidas de mesma letra na colu na não diferem estatisticamente entre si pelo teste t a $10 \%$ de probabilidade.

$\stackrel{1}{ }$ Carfentrazone $=$ carfentrazone-ethyl.

Tabela 3 - Efeito de diferentes herbicidas sobre Commelina villosa, aplicados em pós -emergência tardia. Botucatu-SP

\begin{tabular}{|c|c|c|c|c|c|c|c|c|c|}
\hline \multirow[t]{2}{*}{ Tratamento } & \multirow{2}{*}{$\begin{array}{c}\text { Dose } \\
\left(\mathrm{g} \mathrm{ha}^{-1}\right)\end{array}$} & \multicolumn{7}{|c|}{$\begin{array}{c}\text { Controle }(\%) \\
\text { Dias após a Aplicação }\end{array}$} & \multirow{2}{*}{$\begin{array}{c}\text { Massa } \\
\text { Seca } \\
(\mathrm{g})\end{array}$} \\
\hline & & 1 & 3 & 6 & 9 & 14 & 21 & 28 & \\
\hline Testemunha & - & $0,0 \mathrm{~d}$ & $0,0 \mathrm{~d}$ & $0,0 \mathrm{~d}$ & $0,0 \mathrm{e}$ & $0,0 \mathrm{~d}$ & $0,0 \mathrm{~d}$ & $0,0 \mathrm{c}$ & $8,8 \mathrm{a}$ \\
\hline Carfentrazone $^{\mathrm{L}}$ & 30 & $53,7 \mathrm{~b}$ & $70,0 \mathrm{~b}$ & $70,0 \mathrm{~b}$ & $83,2 \mathrm{~b}$ & $65,5 \mathrm{~b}$ & $61,2 \mathrm{~b}$ & $35,0 \mathrm{~b}$ & $1,4 \mathrm{bc}$ \\
\hline Carfentrazone & 50 & $63,7 \mathrm{a}$ & $87,5 \mathrm{a}$ & $93,7 \mathrm{a}$ & $97,5 \mathrm{a}$ & $100,0 \mathrm{a}$ & $100,0 \mathrm{a}$ & $100,0 \mathrm{a}$ & $0,0 \mathrm{~d}$ \\
\hline Carfentrazone+glyphosate & $30+960$ & $71,2 \mathrm{a}$ & $86,2 \mathrm{a}$ & $92,5 \mathrm{a}$ & $95,0 \mathrm{a}$ & $99,2 \mathrm{a}$ & $100,0 \mathrm{a}$ & $100,0 \mathrm{a}$ & $0,0 \mathrm{~d}$ \\
\hline Sulfentrazone+glyphosate & $150+960$ & $41,2 \mathrm{c}$ & $47,5 \mathrm{c}$ & $47,5 \mathrm{c}$ & $65,0 \mathrm{c}$ & $45,0 \mathrm{c}$ & $38,7 \mathrm{c}$ & $14,0 \mathrm{bc}$ & $2,4 c$ \\
\hline Glyphosate & 960 & $0,0 \mathrm{~d}$ & $0,1 \mathrm{~d}$ & $1,0 \mathrm{~d}$ & $15,0 \mathrm{~d}$ & $36,2 \mathrm{c}$ & $69,5 \mathrm{~b}$ & $96,0 \mathrm{a}$ & $0,5 \mathrm{~cd}$ \\
\hline $\mathrm{F}$ & & $62,91 * *$ & $205,06^{* *}$ & $210,01 * *$ & $84,56 * *$ & $31,27 * *$ & $21,02 * *$ & $26,09 * *$ & $35,87 * *$ \\
\hline $\mathrm{CV}(\%)$ & & 20,62 & 11,58 & 11,54 & 15,40 & 24,07 & 27,12 & 31,66 & 51,87 \\
\hline d.m.s. & & 9,69 & 6,89 & 7,19 & 11,20 & 17,02 & 20,48 & 22,35 & 1,39 \\
\hline
\end{tabular}

** significativo a $1 \%$ de probabilidade.

Médias seguidas de mesma letra na coluna não diferem estatisticamente entre si pelo teste t a $10 \%$ de probabilidade.

${ }^{1}$ Carfentrazone $=$ carfentrazone-ethyl.

em C. benghalensis e C. diffusa, submetidas à aplicação de glyphosate, a ocorrência de regiões cloróticas e áreas necrosadas dispersas pela superficie foliar, com conseqüente queda de folhas.

Com relação aos valores médios de massa seca acumulada pelas plantas das quatro espécies de Commelina estudadas (Tabelas 2 a 5), nota-se que as plantas de C. erecta (Tabela 5) tratadas com glyphosate isolado tenderam a acumular mais biomassa que a testemunha, indicando que houve estímulo no crescimento das plantas tratadas com o herbicida. Cartwright (1976) menciona que, quando o herbicida é aplicado em dose subletal, pode ocorrer estímulo de crescimento e aumento de massa seca. É possivel que a dose comercial aplicada não seja aquela recomendável para $C$. erecta; por isso, a aplicação do herbicida provocou o efeito inverso sobre essas plantas daninhas. Deve-se destacar também que nas plantas submetidas 
Tabela 4 - Efeito de diferentes herbicidas sobre Commelina diffusa, aplicados em pós -emergência tardia. Botucatu -SP

\begin{tabular}{|c|c|c|c|c|c|c|c|c|c|}
\hline \multirow[t]{2}{*}{ Tratamento } & \multirow{2}{*}{$\begin{array}{c}\text { Dose } \\
\left(\mathrm{g} \mathrm{ha}^{-1}\right)\end{array}$} & \multicolumn{7}{|c|}{$\begin{array}{c}\text { Controle }(\%) \\
\text { Dias após a Aplicação }\end{array}$} & \multirow{2}{*}{$\begin{array}{c}\text { Massa } \\
\text { Seca } \\
\text { (g) }\end{array}$} \\
\hline & & 1 & 3 & 6 & 9 & 14 & 21 & 28 & \\
\hline Testemunha & - & $0,0 \mathrm{c}$ & $0,0 \mathrm{c}$ & $0,0 \mathrm{c}$ & $0,0 \mathrm{c}$ & $0,0 \mathrm{c}$ & $0,0 \mathrm{c}$ & $0,0 \mathrm{~d}$ & $12,3 \mathrm{a}$ \\
\hline Carfentrazone $\mathrm{e}^{1 /}$ & 30 & $7,2 \mathrm{~b}$ & $20,0 \mathrm{~b}$ & $30,0 \mathrm{~b}$ & $34,2 \mathrm{~b}$ & $32,5 b$ & $38,5 \mathrm{~b}$ & $33,0 \mathrm{c}$ & $6,1 \mathrm{~b}$ \\
\hline Carfentrazone & 50 & $24,5 \mathrm{a}$ & $33,0 \mathrm{a}$ & $42,0 \mathrm{a}$ & $55,7 \mathrm{a}$ & $53,2 \mathrm{a}$ & $52,0 \mathrm{ab}$ & $49,7 \mathrm{~b}$ & $4,0 \mathrm{~b}$ \\
\hline Carfentrazone+glyphosate & $30+960$ & $23,0 \mathrm{a}$ & $36,7 \mathrm{a}$ & 45,7 a & $52,5 \mathrm{a}$ & $61,2 \mathrm{a}$ & $68,7 \mathrm{a}$ & $75,0 \mathrm{a}$ & $5,0 \mathrm{~b}$ \\
\hline Sulfentrazone+glyphosate & $150+960$ & $3,5 \mathrm{bc}$ & $3,5 \mathrm{c}$ & $6,5 \mathrm{c}$ & $7,7 \mathrm{c}$ & $9,5 \mathrm{c}$ & $12,0 \mathrm{c}$ & $10,7 \mathrm{~d}$ & $12,1 \mathrm{a}$ \\
\hline Glyphosate & 960 & $0,0 \mathrm{c}$ & $2,5 \mathrm{c}$ & $2,0 \mathrm{c}$ & $5,0 \mathrm{c}$ & $5,0 \mathrm{c}$ & $10,0 \mathrm{c}$ & $10,0 \mathrm{~d}$ & $11,6 \mathrm{a}$ \\
\hline $\mathrm{F}$ & & $15,48 * *$ & $25,65 * *$ & $16,80 * *$ & $16,16 * *$ & $13,94 * *$ & $14,71 * *$ & $23,80 * *$ & $10,88 * *$ \\
\hline $\mathrm{CV}(\%)$ & & 58,71 & 40,36 & 47,70 & 47,92 & 51,98 & 46,84 & 39,44 & 29,28 \\
\hline d.m.s. & & 6,99 & 7,90 & 12,40 & 15,20 & 17,16 & 17,35 & 14,39 & 3,01 \\
\hline
\end{tabular}

** significativo a $1 \%$ de probabilidade.

Médias seguidas de mesma letra na coluna não diferem estatisticamente entre si pelo teste t a $10 \%$ de probabilidade.

${ }^{1 /}$ Carfentrazone $=$ carfentrazone -ethyl.

Tabela 5 - Efeito de diferentes herbicidas sobre Commelina erecta, aplicados em pós-emergência tardia. Botucatu-SP

\begin{tabular}{|c|c|c|c|c|c|c|c|c|c|}
\hline \multirow[t]{2}{*}{ Tratamento } & \multirow{2}{*}{$\begin{array}{c}\text { Dose } \\
\left(\mathrm{g} \mathrm{ha}^{-1}\right)\end{array}$} & \multicolumn{7}{|c|}{$\begin{array}{c}\text { Controle }(\%) \\
\text { Dias após a Aplicação }\end{array}$} & \multirow{2}{*}{$\begin{array}{c}\text { Massa } \\
\text { Seca } \\
(\mathrm{g})\end{array}$} \\
\hline & & 1 & 3 & 6 & 9 & 14 & 21 & 28 & \\
\hline Testemunha & - & $0,0 \mathrm{c}$ & $0,0 \mathrm{c}$ & $0,0 \mathrm{~d}$ & $0,0 \mathrm{c}$ & $0,0 \mathrm{c}$ & $0,0 \mathrm{c}$ & $0,0 \mathrm{c}$ & $3,9 \mathrm{ab}$ \\
\hline Carfentrazone $e^{1 /}$ & 30 & $16,2 \mathrm{ab}$ & $27,5 \mathrm{ab}$ & $31,7 \mathrm{~b}$ & $35,0 \mathrm{~b}$ & $22,0 \mathrm{~b}$ & $14,0 \mathrm{bc}$ & $5,7 \mathrm{bc}$ & $2,7 \mathrm{bc}$ \\
\hline Carfentrazone & 50 & $18,0 \mathrm{a}$ & $36,2 \mathrm{a}$ & $56,7 \mathrm{a}$ & $67,7 \mathrm{a}$ & $64,5 \mathrm{a}$ & $43,7 \mathrm{a}$ & $18,7 \mathrm{~b}$ & $1,4 \mathrm{c}$ \\
\hline Carfentrazone+glyphosate & $30+960$ & $9,7 \mathrm{~b}$ & $17,7 \mathrm{~b}$ & $23,7 \mathrm{bc}$ & $34,2 b$ & $40,7 \mathrm{~b}$ & $50,7 \mathrm{a}$ & $65,0 \mathrm{a}$ & $2,4 \mathrm{bc}$ \\
\hline Sulfentrazone+glyphosate & $150+960$ & $2,2 \mathrm{c}$ & $3,7 \mathrm{c}$ & $15,5 \mathrm{c}$ & $22,5 b$ & $25,5 \mathrm{~b}$ & $24,2 \mathrm{~b}$ & $14,5 \mathrm{bc}$ & $3,0 \mathrm{bc}$ \\
\hline Glyphosate & 960 & $0,0 \mathrm{c}$ & $0,0 \mathrm{c}$ & $0,0 \mathrm{~d}$ & $0,0 \mathrm{c}$ & $2,7 \mathrm{c}$ & $5,0 \mathrm{bc}$ & $5,0 \mathrm{bc}$ & $6,1 \mathrm{a}$ \\
\hline $\mathrm{F}$ & & $8,09 * *$ & $13,81 * *$ & $12,47 * *$ & $9,78 * *$ & $9,95 * *$ & $6,92 * *$ & $14,23 * *$ & $2,84 * *$ \\
\hline $\mathrm{CV}(\%)$ & & 74,29 & 58,40 & 57,22 & 61,37 & 59,24 & 68,46 & 69,85 & 58,65 \\
\hline d.m.s. & & 7,02 & 10,17 & 14,94 & 20,00 & 18,83 & 19,27 & 15,56 & 2,35 \\
\hline
\end{tabular}

** significativo a $1 \%$ de probabilidade.

Médias seguidas de mesma letra na coluna não diferem estatisticamente entre si pelo teste t a $10 \%$ de probabilidade.

1/ Carfentrazone $=$ carfentrazone-ethyl.

ao tratamento sulfentrazone + glyphosate (150 + $960 \mathrm{~g} \mathrm{ha}^{-1}$ ) foram observados sintomas como desfolhamento parcial no caule e necrose apenas nas folhas dos ponteiros, sem comprometimento do desenvolvimento das plantas que rebrotaram após alguns dias, pois os ramos primários e secundários permaneceram intactos e verdes.

Dos tratamentos avaliados, carfentrazoneethyl + glyphosate $\left(30+960 \mathrm{~g} \mathrm{ha}^{-1}\right)$ foi o que apresentou o melhor resultado para controlar as espécies daninhas de Commelina (Tabelas 2 a 5), embora o controle de C. diffusae C. erecta não tenha ultrapassado 75 e $65 \%$, respectivamente (Tabelas 4 e 5). Ressalta-se ainda que a identificação precisa da espécie tratada deve ser considerada, uma vez que $C$. diffusa e C. erecta apresentaram controle insatisfatório, enquanto $C$. benghalensis e C. villosa foram controladas eficientemente. Ronchi et al. (2002) também verificaram que, dentre os 
tratamentos testados para controlar C. diffusa e C. benghalensis, a utilização da mistura carfentrazone-ethyl com glyphosate foi mais eficiente no controle dessas trapoerabas.

Embora tenha sido verificado que a mistura de sulfentrazone + glyphosate não apresenta bons resultados (Tabelas 2 a 5), de certa forma os resultados obtidos corroboram aqueles já constatados por Tollervey et al. (1979), Galli (1991), Ramos \& Durigan (1996), Ronchi et al. (2002) e Werlang \& Silva (2002), ou seja, que a aplicação de glyphosate em mistura com outro herbicida resulta no melhor tratamento para controle de trapoerabas. O mecanismo de translocação do herbicida, provavelmente, é relevante para explicar esse fato, já que o sulfentrazone é um herbicida de contato e translocado principalmente pelo xilema, enquanto carfentrazone-ethyl e glyphosate são ambos sistêmicos e translocados preferencialmente pelo floema (Rodrigues \& Almeida, 2005). Assim, deve ter ocorrido sinergismo entre os dois herbicidas com o mesmo mecanismo de translocação, acelerando e acentuando as injúrias provocadas por cada um deles isoladamente (Tollervey et al., 1979).

Portanto, pode-se afirmar que o controle químico das trapoerabas estudadas foi dependente da espécie, sendo $C$. benghalensis e C. erecta, respectivamente, as espécies mais e menos controladas com os tratamentos testados. A utilização de carfentrazone-ethyl + glyphosate foi mais eficiente no controle dessas plantas daninhas.

\section{LITERATURA CITADA}

BARRETO, R. C. Levantamento das espécies de Commelinaceae R. Br. nativas do Brasil. 1997. 490 f. Tese (Doutorado em Botânica) - Universidade de São Paulo, São Paulo, 1997.

CARTWRIGHT, P. M. The history and classification of herbicides. In: AUDUS, L. J. (Ed.) Herbicides - physiology, biochemistry, ecology. 2. ed. London: Academic Press, 1976. v. 1. p. 55-82.

CARVALHO, J. E. B. et al. Avaliação da eficiência do produto 2,4-D + glyphosate no controle de plantas daninhas na cultura de cacau. In: CONGRESSO BRASILEIRO DE HERBICIDAS E PLANTAS DANINHAS, 18., 1991, Brasília. Resumos... Brasília: SBHED, 1991. p. 95-96.

Planta Daninha, Viçosa-MG v. 25, n. 2, p. 359-364, 2007
DE LA VEGA, M. H. et al. Control de Commelina erecta L. con herbicidas postemergentes com el objectivo de su uso en cultivo de soja transgénica. Planta Daninha, v. 18, p. 51-56, 2000 .

GALLI, A. J. B. Avaliação da eficiência de glyphosate em diversos produtos no controle de Commelina virginica (trapoeraba) em citros. In: CONGRESSO BRASILEIRO DE HERBICIDAS E PLANTAS DANINHAS, 18., 1991, Brasília. Resumos... Brasília: SBHED, 1991. p. 104-105.

LORENZI, H. Manual de identificação e controle de plantas daninhas. 6. ed. Nova Odessa: Instituto Plantarum, 2006. 339 p.

MONQUERO, P. A.; CURY, J. C.; CHRISTOFFOLETI, P. J. Controle pelo glyphosate e caracterização geral da superfície foliar de Commelina benghalensis, Ipomoea hederifolia, Richardia brasiliensis e Galinsoga parviflora. Planta Daninha, v. 23, p. 123-132, 2005.

PENCKOWSKI, L. H.; ROCHA, D. C. Guia Ilustrado de identificação e controle de espécies de trapoerabas. Castro: Fundação ABC, 2006. 40 p.

PIMENTEL-GOMES, F. Curso de estatística experimental. 6. ed. Piracicaba: Binetti, 1976. 430 p.

RAMOS, H. H.; DURIGAN, J. C. Avaliação da eficiência da mistura pronta de glyphosate $+2,4$-D no controle da Commelina virginica L. em citros. Planta Daninha, v. 14, p. 33-41, 1996.

ROCHA, D. C.; RODELLA, R. A.; MARTINS D. Ocorrência de Commelina villosa como planta daninha em áreas agrícolas no Estado do Paraná-PR, Brasil. Planta Daninha, v. 18, p. 161-167, 2000.

RODRIGUES, B. N.; ALMEIDA, F. S. Guia de herbicidas. 5. ed. Londrina: IAPAR, 2005. 591 p.

RONCHI, C. P. et al. Misturas de herbicidas para o controle de plantas daninhas do gênero Commelina. Planta Daninha, v. 20, p. 311-318, 2002.

SOCIEDADE BRASILEIRA DA CIÊNCIA DAS PLANTAS DANINHAS - SBCPD. Procedimentos para instalação, avaliação e análise de experimentos com herbicidas. Londrina: 1995.42 p.

TOLLERVEY, F. E. et al. Weed control investigations in Bolivian crops 1977-1978. Weed Abstract, v. 29, p. 225, 1979.

TUFFI SANTOS, L. D. et al. A. Efeito do glyphosate sobre a morfoanatomia das folhas e do caule de Commelina diffusa e C. benghalensis. Planta Daninha, v. 22, p. 101-107, 2004.

WERLANG, R. C.; SILVA, A. A. Interação de glyphosate com carfentrazone-ethyl. Planta Daninha, v. 20, p. 93-102, 2002.

WILSON, A. K. Commelinaceae - a review of the distribution, biology and control of the important weeds belonging to this family. Trop. Pest Manag., v. 27, p. 405-418, 1981. 